\title{
EMERGING TRENDS IN PERVASIVE COMPUTING ARCHITECTURE FOR BIG DATA ANALYTICS IN MOBILE DEVICE
}

\author{
S.Sangeetha \\ Department of CS \\ Dharmapuram Adhinam Arts College, \\ Mayiladuthrai Tamil Nadu, India.
}

\begin{abstract}
The data from the major challenge in mobile devices because of ever present of various kinds of sensors. The Big data can be processed by analytics using different tools for the R-Programming, Python, SAS, and Tableau Public. So, we can get useful information in pervasive computing architecture. The data generated for sensor network in smart devices like mobile laptop and other devices. Data analytics is difficult with limited process power. Tableau public tool with good connectivity to cloud can provide us some data analysis tools. Big data can help in many ways which will guarantee better services and openness is lacking in most of the devices. In this paper we have captured the twitter through live barometer and an amorphous form with the help of apache Flume, which how to fetch data from twitter service and store it in HDFs using tools for Tableau public. Those tools can be useful and decision making for us.
\end{abstract}

Keywords - Apache Flume, Barometer, Big Data, Pervasive Computing, sensor network, Tableau Public.

\section{INTRODUCTION}

Big data is like to small data then having data bigger it needs different methods procedures utensils and style. Big data makes value from the storage and processing of very large quantities of digital information. This have a 3 characteristics of big data analysis of data volume, data velocity and data variety.

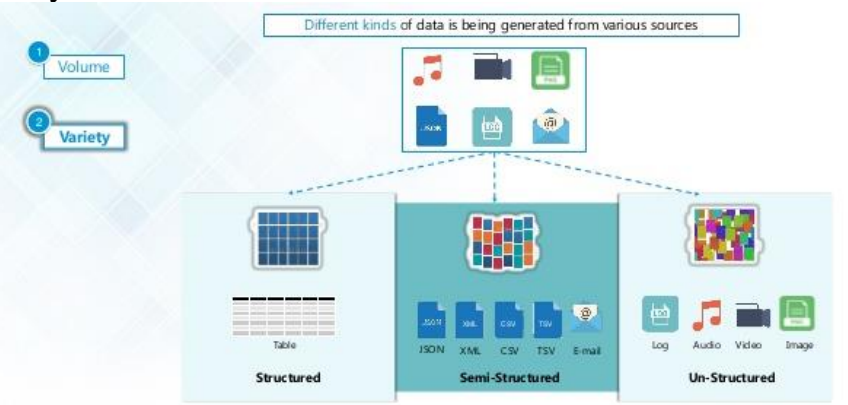

Fig 1. Big Data Structure

\author{
G.Deepalakshmi \\ Department of CS \\ Dharmapuram Adhinam Arts College, \\ Mayiladuthrai, Tamil Nadu, India
}

Barometer an instrument measuring atmospheric pressure which used to help of forecast the weather by measuring air. This can measure the pressure by the atmosphere using water, air and mercury. Tableau is a expert Aptitude tool for visually analyzing the data. Users can create and issue an interactive and shareable dashboard, which depict hetrends, variations, and density of the data in the form of graphs and charts. These three steps are

- Connect to a data foundation - It involves locating the data and using an appropriate type of connection to read the data.

- Choose scopes and actions - This involves selecting the required columns from the source data for analysis.

- Apply conception technique - This involves applying required visualization methods, such as a specific chart or graph type to the data being analyzed.

\section{BAROMETER IN MOBILE ARCHITECTURE}

A. Flume Architecture

Flume has a flexible design based upon gushing data flows. It is fault easygoing and vigorous with recovery instruments. Flume has different levels of dependability to offer which includes 'best-effort delivery' and an 'end-to-end delivery'. Best-effort-delivery. Flume has its own query processing engine which makes it easy to transform each new batch of data before it is moved to the intended sink. Flume Load data from Twitter using Flume:

Step 1) Go to the manualholdingbasis code documents in it.

Step 2) Set CLASSPATH to contain <Flume Installation Dir $>/$ lib/* and $\sim$ FlumeTutorial/flume/mytwittersource/*

Step 3) Compile basis code using the command. 


\section{International Journal of Engineering Applied Sciences and Technology, 2020 Vol. 5, Issue 1, ISSN No. 2455-2143, Pages 586-590 \\ Published Online May 2020 in IJEAST (http://www.ijeast.com)}

A barometer is an unreceptive sensor (rather than an "energetic" sensor, like GPS). An app could attentive you to your elevation when you method certain beginnings - short of sucking down your mobile like GPS does. Perhaps apps like My Tracks could be updated to include an "altitude

trigger" option, which could turn on the GPS to record your location at 50-foot increments of promotion change, saving your batter. Nevertheless silent recording a rather accurate track.

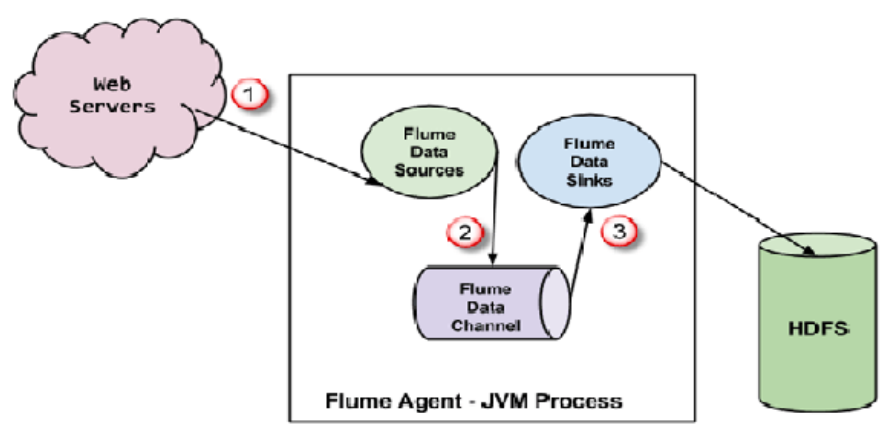

Fig 2. Flume Architecture

\section{B. Tableau Architecture}

Data Source: Tableau can connect to and also mix together the data from multiple data sources. It can connect to data sources remotely. It still, it can connect to an Excel file, a web application or a database.

Data Connectors: Data Connectors consist of Tableau Engine Data Engine in Tableau: Data Engine stores the tableau data extract which is pooled from various data sources which responses to any visualization that has been created using the tableau data extract in a database. The job of the data engine to process the various requests coming to the tableau data extract. The data engine gets VizQL Server which can the user is directly connecting to any database.

ODBC/sql connector: It can extract both live as well as inmemory data depending on the requirement. A feature of the ODBC connector is it can connect to any databases without using their native connector.

Live data: Tableau can provide live data by connecting to external data sources using the existing infrastructure of their databases by sending dynamic MDX and sql statements.

In-memory data: We can also make a copy of the extracted data from external sources in the form of tableau data extracted from the file.

Application server: It is a server process that executes everything but visualization in Tableau. It does content management, authentication processes in tableau. It is multiserver, multi-process, and multi-threaded.

VizQL Server: It is the heart of Tableau's process and the most important as it is what makes Tableau unique. So the request data comes to this server and converts the request into an SQL query and then sends the query directly to the data source using respective data source drivers. The requested data comes back to the VizQL server where it is processed and data is sent in the form of images that are then displayed to the front end. It is multi-server, multi-process, and multi-threaded.

Data Server: It serves as a proxy for data sources. It is by using this proxy that tableau is able to connect to databases. One of its key features is that it provides metadata management for data sources.

Gateway: Gateway is a very much light weighted apache web server. So, whenever a client tries to for example login to the tableau server. The gateway, in this case, will forward the request to the application server for authentication purposes.

So it is the gateway where we feed the request and it carries it to different processes depending on the type of request. At the end of your request processing, the gateway reassembles the HTML response and shows it on the client browser. If no external load balancing is present, the gateway will also load the balancing of some of the requests routed between distinct procedures. One important point to note is that gateway is a multi-server single process and multi-threaded which means we cannot have multiple instances of gateway running in them.

Clients: This is the User Interface from where we send the requests and all the visualizations can be viewed or edited from here.

Tableau Desktop: : it's associate analytical tool which will facilitate to make and think about dashboards in Tableau Server using Tableau Desktop.

Web: Just like mobile browsers many web browsers like Google Chrome, Internet Explorer, Safari support the Tableau Server and visualizations in the dashboard can be viewed and abbreviated using these browsers.

salient features of Tableau are mentioned.

- live data from external databases for our visualizations by directly connecting them.

- The visualizations can be viewed using different clients: Tableau Desktop, Mobile and Web.

- $\quad$ interactive dashboards.

- list of native data connectors.

- share dashboards with Tableau.

- Mobile ready dashboards.

- Tableau server translates data queries into visualizations. 

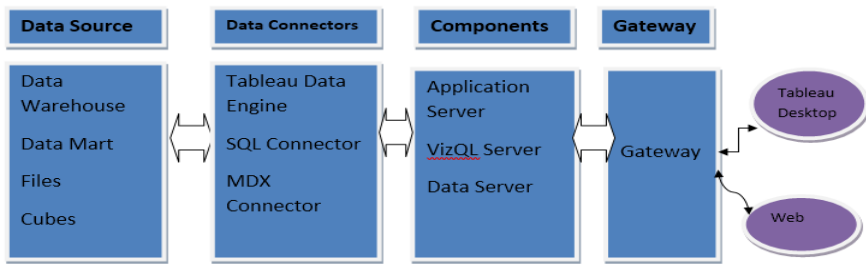

Fig 3. Tableau Architecture

pervasive computing is the result of computer technology advancing at exponential speeds -- a trend toward all manmade and some natural products having hardware and software. The goal of pervasive computing, which combines current network technologies with wireless computing, voice recognition, Internet capability and artificial intelligence, is to create an environment where the connectivity of devices is embedded in such a way that the connectivity is unobtrusive and always available.

\section{ENHANCED BAROMETER ASOS}

\section{EXPERIMENT AND RESULT}

The new generation of sensors uses sound waves to calculate wind speed and direction. The measurement is based on the time it takes for an ultrasonic pulse to travel from one transduction to another, which varies depending on among other factors - the wind speed. The passage time is measured in both directions for several (usually two or three) pairs of the transducer heads. Based on those results, the sensor computes wind pace and direction. The mechanical sensors, the ultrasonic sensors to be compared suggest several rewards such as no moving parts, advanced self-diagnostic capabilities and reduced maintenance requirements.

NWS and FAA ASOS stations and most of new AWOS installations are at present ready with ultrasonic wind sensors.

Different all other measurements, which are made between 3 and 9 feet (1 and 3 meters) above the ground, wind speed and direction are measured at 30 feet (10 meters).

mechanized airport weather stations use a light emitting diode weather identifier (LEDWI) to determine if and what type of precipitation is falling. The LEDWI sensor dealings with glittering pattern of the precipitation declining through the sensor's infrared beam (approximately 50 millimeters in diameter) and determines from a pattern analysis of the particle size and fall rapidity whether the precipitation is rain or snow.

If rainfall is determined to be falling, but the pattern is not conclusively identified as either rain or snow, unknown precipitation is reported. Automated airport weather stations are not yet able to report hail, ice pellets, and various other inbetween forms of precipitation

\section{A. Calucutate barometric pressure}

The pressure is also referred to as the Assume a barometer with

a cross-sectional area $L$,

a height $X$, filled with mercury from the bottom at Point $S$ to the top at Point $T$. The pressure at the bottom of the barometer, Point $\mathrm{S}$ is equal to the atmospheric pressure.

The pressure at the very top, Point T, can be taken as zero. Since there is only mercury vapor above this point and its pressure is very low-slung relative to the atmospheric pressure. So, one can find the atmospheric pressure using the barometer and this equation.

Where o is the density of mercury,

$\mathrm{r}$ is the gravitational acceleration

$\mathrm{x}$ is the height of the mercury column above the free outward area. The physical dimensions of the barometer itself have no effect on the height of the fluid column in the tube.

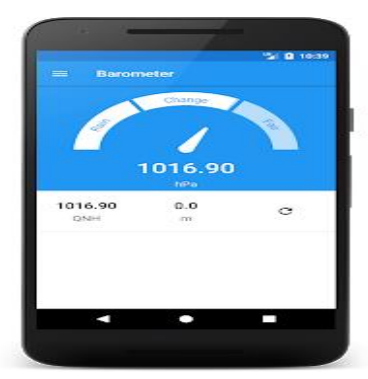

Fig 4. Barometer in mobile

\section{B. Improved Meter algorithm}

METAR is a format for reporting weather information. A METAR weather report is for the most part of used by aircraft pilots, besides by meteorologists, who use combined METAR information to contribution in weather forecasting.

Fresh METAR is the transmission of observational weather data. It is highly consistent through the International Civil Aviation Organization (ICAO), which permits it to be understood throughout most of the world.

RVR is used as one of the main criteria for minima on instrument methods, as in most cases a pilot must find graphic reference of the airstrip to land an aircraft. 


\section{International Journal of Engineering Applied Sciences and Technology, 2020 Vol. 5, Issue 1, ISSN No. 2455-2143, Pages 586-590 \\ Published Online May 2020 in IJEAST (http://www.ijeast.com)}

The maximum RVR range is 2,000 meters or 6,000 feet, above which it is not important, thus does not need to be reported. RVRs are providing in METARs

RVR is used as one of the main criteria for minima on instrument methods, as in most cases a pilot must find graphic reference of the airstrip to land an aircraft.

The maximum RVR range is 2,000 meters or 6,000 feet, above which it is not important, thus does not need to be reported. RVRs are providing in METARs

That is transmitted by air traffic controllers to aircraft making methods to allow pilots to assess whether it is practical and permissible to make an method.

RVR is used as one of the main criteria for minima on instrument methods, as in most cases a pilot must find graphic reference of the airstrip to land an aircraft

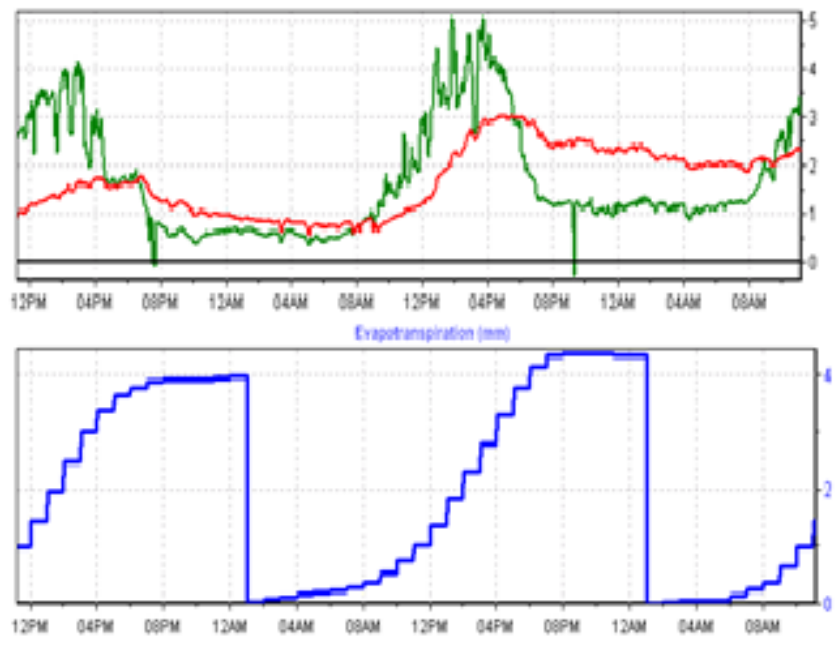

Fig 5. Wave for Wind strengths using RVR

. The maximum RVR range is 2,000 meters or 6,000 feet, above which it is not important, thus does not need to be reported. RVRs are providing in METARs

That are transmitted by air traffic controllers to aircraft making methods to allow pilots to assess whether it is practical and permissible to make an method.

RVR is also the main criterion used to control the category of graphic aids that are connected at an airport. The International Civil Aviation Organization ICAO stipulate in its Annex 14 that for RVR values above $550 \mathrm{~m}$,

Phase I : lighting be going to be installed, if RVR is between $300 \mathrm{~m}$ and $549 \mathrm{~m}$ then

Phase II: lighting is compulsory.

Phase III a:connected for RVR values between $175 \mathrm{~m}$ and $300 \mathrm{~m}$.
PhaseIII b: mandatory for RVR values between $50 \mathrm{~m}$ and $175 \mathrm{~m}$ though there is no

Phase IIIc : RVR restriction graphic aids.

\section{CONCLUSION}

The presented article clearly demonstrates the huge possibilities of Tableau Public software in the area of Pesticide Science and Education. Using the software public with ease of forecast report specially wind direction in our mobile device. There properties revealing their effectiveness, safety and correct use under given conditions and circumstances by summarizing.

The completely Visualization and extremely easy to be use interface of the Tableau Public make it android mobile device for the education of the Weather report, while the more complex software as Microsoft Access or SQL is more suitable for thus any situation

Tableau public in this aspect can be used as from weather report as from aircraft, surface and other things to specialists and creation of decision supporting infotopias for the right and correct Travel area used in tour. The availability of the Android version of the software is a big advance for this.

Tableau has intermediate position as decision supporting tool between software for electronically tables and relational databases definitely more suitable for users (specialists) with less computer software skills.

Although, Microsoft Excel or Open Office Calc also have similar properties in the face of Dash board, Tableau provide from one side much more easy to be understand and use interface, and from the another - much more possibilities for combinations and graphical presentations of the data.

\section{REFERENCE}

[1] Knowles Middleton W. E et al "Brief History of the Barometer". Barometer. ws. Archived from the originalon 14January 2010. Retrieved 2010-02-04.

[2] Schuol, J. and Abbaspour, K.C.et al., (2007) Using Monthly Weather Statistics to Generate Daily Data in a SWAT Model Application to West Africa. Ecological Modelling, 201, 301-311.

[3] Healey, Ramaswamy et al., Visualizing twitter sentiment, ps://www.csc2.ncsu.edu/faculty/healey/tweet_viz/, 2016

[4] Tableau Software: Build your big data platform with Tableau and Cloudera (2018).. Accessed 10 Mar 2019

[5] T. A. Keahey, et al., Using visualization to understand big data, Technical Report, IBM Corporation, 2013, pp. $1-16$. 
[6] B. Porter,et al., Visualizing Big Data in Drupal: Using Data Visualizations to Drive Knowledge Discovery, Report, University of Washington, October 2012, pp. 138.

[7] Marr, Bernard, et al., "What Is Big Data? A Super Simple Explanation for Everyone.”, 2012. URL

[8] Duong, Van minh, et al., Sentiment and Influence Analysis of Twitter Tweets, United States (12) Patent Application Publication (10) Pub. N0.: US 2013/0103667 A1 Minh (43) Pub. Date: Apr. 25, 2013

[9] SUBJ, et al., Automated Weather Observing Systems (AWOS) for Non-Federal Applications - with change 1 Document Information". Faa.gov. Retrieved 23 May 2019.

[10] Julian M. Wright JR, et al., Surface Weather Observation Stations - ASOS/AWOS". Faa.gov. Retrieved 23 May2019

[11] SUBJ et al., Receipt and Dissemination of Weather Observations" (PDF). Faa.gov. Retrieved 23May 2019.

[12] Jain and Bagree, et al., "A prepaid meter using mobile communication", International Journal of Engineering, Science and 164 Technology, Vol. 3, No. 3, 2011, pp. $160-166$.

[13] R. Grimm, T. Anderson, B. Bershad, and D. Wetherall. et al., A system architecture for pervasive computing. In Proceedings of the 9th ACM SIGOPS European Workshop, pages 177- 182, Kolding, Denmark, Sept. 2000.

[14] Lalana Kagal, Vlad Korolev, Harry Chen, Anupam Joshi, and Tim Finine. et al., Centaurus: A framework for intelligent services in a mobile environment. In Proceedings of International Workshop on Smart Appliances and Wearable Computing (IWSAWC), in the 21st International Conference on Distributed Computing Systems (ICDCS-21) April 16-19, 2001.

[15] Banavar, G. et al., "Challenges: An Application Model for Pervasive Computing", Proceedings 6th Annual Intl. Conference on Mobile Computing and Networking (MobiCom 2000), August 2000. 